
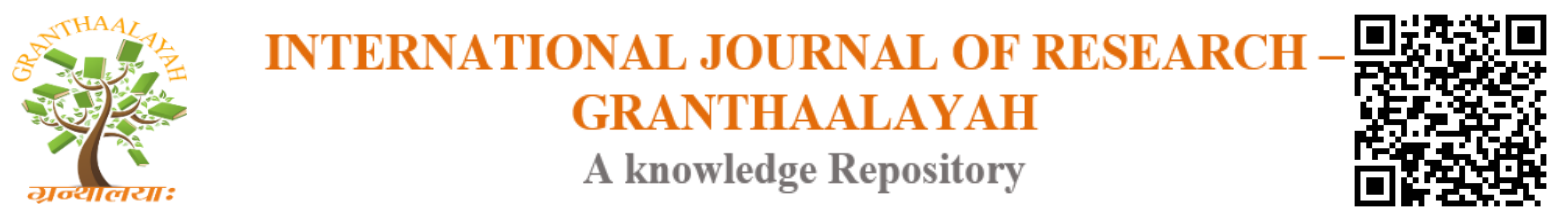

Science

\title{
AN AYURVEDIC APPROACH IN THE MANAGEMENT OF JALODAR (ASCITES): A CASE STUDY
}

\author{
Dr. Dnyaneshwar. K. Jadhav ${ }^{1}$, M.D. Kaychikitsa (Ayurved) ${ }^{1}$ \\ ${ }^{1}$ Assistance Professor, Kaychikitsa Department, Shri Dhanwantri Ayurvedic Medical College \\ \& Research Centre 281401, India
}

\begin{abstract}
"AKRIYAYAM DHRUWO MRUTYU, KRIYAYAM SHANSHYO BHAWET" this is interesting statement regarding jalodar found in charak samhita. If we are not doing treatment then patient will die and even we do treatment then also patient will die. Udar (acities) is such kind of disease.

Shakespeare very well said that, 'To be or not to be' was my question. Being doctor we are mounting to be care, which may be border of life $\&$ death. In such condition sending back to patient it may be criminal act to doctor. So we must treat the patient until he breath last.

After all Morden technics and medical facilities and development still there is no sure treatment which cure patient totally. In jalodar Tapping and all treatment gives temporary relief. Again fluid gets collected in abdominal cavity. In such case Ayurvedic treatment is the best.

In Present case study, 45 year male patient have been suffering from Udarvriddhi (Increased abdominal girth), Jwaraprachiti (fever), Droubalya (Gen.Weakness), kshudhamandya (decreased appetite), Ubhaypadshoth since 4 month which get relive in 15 days and disappear within 1 month. Ayurvedic management on Jalodar such as herbal drug, Nityavirechana (purgative), Agnideepan (increase appetite), balaprapti, yakrituttejjak, external application of Arka-patta bandhana, only Dugdha Aahar act on root of pathology of Jalodar and by doing Samparptibhanga it give result in Jalodar.
\end{abstract}

Keywords: Jalodar; Acities; Ayurvedic Management.

Cite This Article: Dr. Dnyaneshwar. K. Jadhav, and M.D. Kaychikitsa. (2018). “AN AYURVEDIC APPROACH IN THE MANAGEMENT OF JALODAR (ASCITES): A CASE STUDY." International Journal of Research - Granthaalayah, 6(11), 439-444. https://doi.org/10.29121/granthaalayah.v6.i11.2018.1152.

\section{Introduction}

Jalodar is generalised abdominal enlargement. Madagni (poor Appetites), doshati sanchaya (collection of toxic substance in body), and Paap karma (bad nature) are main cause of jalodar ${ }^{[1]}$. pran-apan-agni dushti are main pathology in Jalodar ${ }^{[2]}$. In modern science we can co-relate it with ascites. 
The word ascites is of Greek origin (askos) and means bag or sacacities describe the condition of pathological fluid collection within the abdominal cavity. ${ }^{[3]}$

"Nityameva Virechayet" This short chikitasa Sutra describe Maharshi Charaka for Jalodar (ascites) ${ }^{[4] .}$

Ayurvedic management such as herbal drug, Nityavirechana (purgative), Agnideepan (Increase appetite), Balaprapti, Yakrituttejjak (stimulant for hepatic function, external application of Arkapatta bandhana (belt made by leaves of Calotropisprocera), only Dugdha Aahar act on root of pathology of Jalodar and by doing Samparptibhanga it give best result in Jalodar . Ayurvedic management still stand test to time.

\section{A case report}

A 45 year old male patient came to us with chief compliant of -

1) udarvriddhi (Increased abdominal girth).

2) Jwaraprachiti (fever).

3) Droubalya (Gen. weakness).

4) Kshudhamandya (Decreased appetite).

5) Ubhaypadshoth (Pedal edema).

Patient had above complaints since 4 months.

H/O Amalpitta.

No H/o Dm / HTN, Asthma

\section{History of Personal Illness}

The patient was normal 4 month back. Since then patient have been suffering from Udarvriddhi (increased abdominal girth), Jwaraprachiti (fever), Droubalya (Gen. weakness), Kshudhamandya (decreased appetite), Ubhaypadshoth ( pedal edema) ${ }^{[5]}$. For this patient took treatment from different modern doctor but got no relief, then he came to our hospital - Seth Sakharam Nemchand Jain Ayurvedic Rugnalaya in Kaychikitsa department opd. For better Management we admitted patient in Ipd.

\section{Personal History}

Occupation: Farmer.

Bad Habits: Chronic alcoholism.

$\mathrm{O} / \mathrm{E}$ :

Nadi $($ pulse $)=80 / \mathrm{min}$.

Mala $($ stool $)=$ Normal.

Mutra $($ urine $)=$ Normal.

Jeeva $($ tounge $)=$ Eshatha saam.

Agni = Kshudhamandya.

Shabda $($ speech $)=$ Normal.

Druka (eyes) $=$ Pallor ++ , icterus +++ .

Akruti $=$ krusha . 
Bala $=$ heena .

Raktadaaba $($ B.P) $=110 / 70 \mathrm{~mm} / \mathrm{Hg}$.

\section{Material and Method}

\section{Material}

Chart.No-1: Showing material for Management of Jalodar as

\begin{tabular}{|c|c|c|c|c|}
\hline SR.NO & DRAVYA & DOSE & DURATION & ANUPANA \\
\hline 1. & Sharapunkaha & $500 \mathrm{mg}$ & \multirow{3}{*}{1 pack TDS } & \multirow[t]{3}{*}{ Luke warm water } \\
\hline 2. & Daruharidra & $1 \mathrm{gm}$ & & \\
\hline 3. & Amalaki & $300 \mathrm{mg}$ & & \\
\hline 6. & Pachak vati & $250 \mathrm{mg}$ & $1 \mathrm{BD}$ & Luke warm water. \\
\hline 7. & Aragyawardhini & $250 \mathrm{mg}$ & $1 \mathrm{BD}$ & Luke warm water. \\
\hline 8. & Pittashekahr rasa & $250 \mathrm{mg}$ & $1 \mathrm{BD}$ & Luke warm water. \\
\hline 9. & Laxmivilaas Guti & $125 \mathrm{mg}$ & $1 \mathrm{BD}$ & Luke warm water. \\
\hline 10. & Punarnavadi kwatha & $2 \mathrm{TSF}$ & Twice in days & Luke warm water. \\
\hline
\end{tabular}

CHART.NO - 2: Showing Panchkarma Management of Jalodar as -

\begin{tabular}{|l|l|}
\hline \multicolumn{2}{|c|}{ PANCHKARMA } \\
\hline Arkapatta Bandhana & Arkapatra + Eranda taila \\
\hline Basti & Triphala + Til tail + Sendhawa \\
\hline Virechana & Abahaydi modak $(1 / 2$ tablet with Triphala Kwath $)$ \\
\hline
\end{tabular}

\section{Method}

- Center of study: S.S.N.J.Ayurvedic Rugnaayan, Solapur.

- Type of study: Simple random single case study.

\section{Discussion}

\section{Discusion On Jalodar (Ascites)}

Ayurvedic term Jalodar Vyadhi is homologous to Ascites described in modern medicine.

According to Ayurveda Chikitsa sutra of Udara choice of treatment is, 'Nitya Virechana' (purgative), Agnidipan (increase appetite), Balaprapti and Yakritottejaka Chikitsa (Stimulating hepatic function) and also Arka pattabandhan over abdomen as external application. Appreciable results were observed in the form of reduction of abdominal girth, bipedal oedema, increase appetite, increase strength.

\section{Hetu of jalodar as ${ }^{[6]}$}

1) Ahar

- Improper and irregular diet Causes the disturbance of vatadosha.

- Jirna Madyapana (Chronic Alcoholism).

- Ati-ruksha, Lawan, Vidahi Annasevan.

2) Vihar 
- Supresion of natural urga (chhardi vega dharan ).

- Ratri jagran.

\section{SAMPRAPTI GHATAK}

CHART.NO-3: Showing samprapti Ghatak of Jalodar as -

\begin{tabular}{|l|l|}
\hline \multirow{2}{*}{ DOSHA } & Vata (Prana, Saman, Apan) \\
\cline { 2 - 2 } & Pitta (Pachak, Sadhak) \\
\hline DUSHYA & Rasa. \\
\hline MALA & Sweda. \\
\hline SROTUS & Ambuvaha, Swedavaha. \\
\hline STANA & Udar \\
\hline
\end{tabular}

\section{SAMPRAPTI}

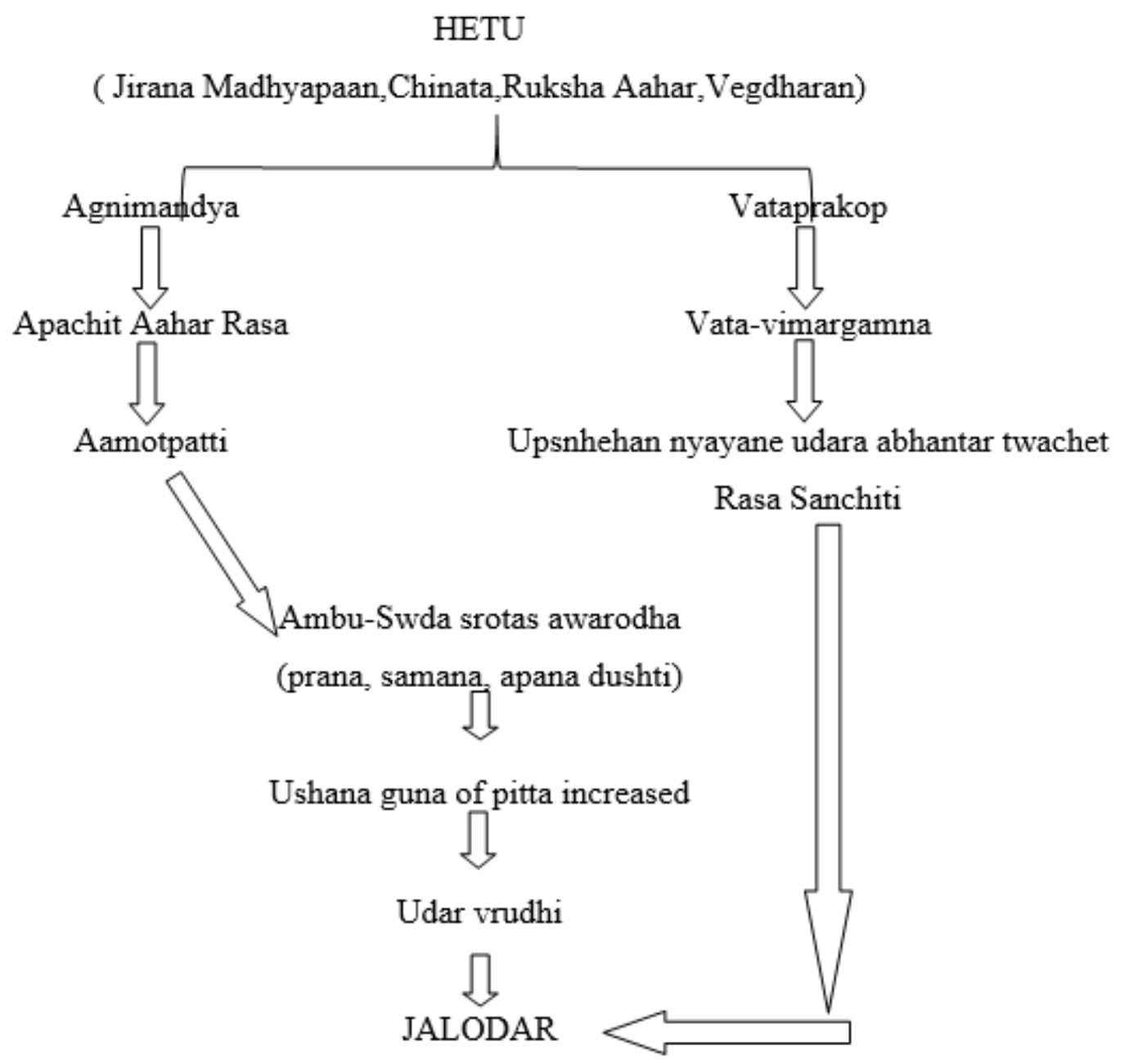

\section{Discusion on Action of Drug and Other Procedure}

- Sharapunkha ${ }^{[7]}$ - yakrutojaak, diapn, pachan.

- Daruharidra ${ }^{[8]}$-Pittaghana, Dipan, Yakruttejak, Kandughana,Vrana. 
- Aamalki [9] Pittaghana, kledaghna,Rasayana,Agnidiapn \& Aampachan.

- Pachak vati ${ }^{[10]}$ - Pacchana mainly Amadosa nasaka \& Agni vardhaka.

- Aarogyavardhini vati ${ }^{[11]}$-Grahanishodhak,Diapan,Pachan,Pakwashyadushti nashka.

- Laxmivilaas Guti ${ }^{[12]}$ - Rasayana, Rudhya, Balya, Krumighna, Vednastapana, Mutral, Pachaka.

- Pittashekahar rasa- Vata-pittghna, virechyia

- Punarnawadi kwatha [13] - Shotaghna,mutral.

- Arka pattabandhana - Arka pattabandhana is avoid Vataprakop due to its Mrudu swedana. It reduced the srotorodh in udar.

- Basti - [ Triphala+ Til tail+ Sendhawa] = for Vatanulomana, Malavibandhanashk, Grahanidoshnashaka

- Virechana: chikitsa sutra of jalodar is - 'Nitya Virechna'. Liver (yakrit) is the mula-sthana of Rakta. Rakta-Pitta has Ashray and Ashraayi sambhnda, hence for elimination of vitiated Pitta Dosha virechan is the best Chikitsa. Virechana also decreases abdominal girth and oedema by decreasing fluid in the abdominal cavity. Here we use tablet.Abahyadi Modak for virechan.

- Aahar ${ }^{[14]}$ : Role of diet also important as equal to medicine. Diet in jalodar (acitis) should be milk (Godugdha) only. Godugdha (Cow Milk) is only complete food which is full of nutrients and easily digestible. With Godugdha we advised patient to take Takrapaan and jwari Roti as Supplimentary food with Godugdha.

\section{Observation and Result}

CHART.NO-4: Showing Abdominal Girth Measurement

\begin{tabular}{|l|l|l|l|}
\hline & Date 8 cm above umbilicus & At umbilicus & $\mathbf{8} \mathbf{c m}$ below umbilicus \\
\hline 24.1 .2015 & $80 \mathrm{~cm}$ & $80.5 \mathrm{~cm}$ & $75.5 \mathrm{~cm}$ \\
\hline 26.1 .2015 & $80 \mathrm{~cm}$ & $80.5 \mathrm{~cm}$ & $75.5 \mathrm{~cm}$ \\
\hline 28.1 .2015 & $80 \mathrm{~cm}$ & $81 \mathrm{~cm}$ & $76 \mathrm{~cm}$ \\
\hline 30.1 .2015 & $78.5 \mathrm{~cm}$ & $78 \mathrm{~cm}$ & $72 \mathrm{~cm}$ \\
\hline 1.2 .2015 & $81 \mathrm{~cm}$ & $82.5 \mathrm{~cm}$ & $74.5 \mathrm{~cm}$ \\
\hline 3.2 .2015 & $81 \mathrm{~cm}$ & $81.5 \mathrm{~cm}$ & $75 \mathrm{~cm}$ \\
\hline 4.2 .2015 & $79 \mathrm{~cm}$ & $78 \mathrm{~cm}$ & $72.5 \mathrm{~cm}$ \\
\hline 5.2 .2015 & $76.5 \mathrm{~cm}$ & $76 \mathrm{~cm}$ & $71 \mathrm{~cm}$ \\
\hline 6.2 .2015 & $75 \mathrm{~cm}$ & $74 \mathrm{~cm}$ & $70 \mathrm{~cm}$ \\
\hline 7.2 .2015 & $70.5 \mathrm{~cm}$ & $69 \mathrm{~cm}$ & $67.5 \mathrm{~cm}$ \\
\hline
\end{tabular}

CHART.NO-5: Showing Table showing improvement Pedal oedema

\begin{tabular}{|l|l|l|}
\hline Date & Just below knee & Just above knee \\
\hline \multirow{2}{*}{24.1 .2015} & Rt. $30 \mathrm{~cm}$ & Rt. $26 \mathrm{~cm}$ \\
\cline { 2 - 3 } & Lt. $31 \mathrm{~cm}$ & Lt. $25 \mathrm{~cm}$ \\
\hline \multirow{2}{*}{30.1 .2015} & Rt.28 cm & Rt $.23 .5 \mathrm{~cm}$ \\
\cline { 2 - 3 } & Lt $.29 \mathrm{~cm}$ & Lt $.23 \mathrm{~cm}$ \\
\hline 7.2 .2015 & Rt. $26 \mathrm{~cm}$ & Rt. $19 . \mathrm{cm}$ \\
\cline { 2 - 3 } & Lt. $25.5 \mathrm{~cm}$ & Lt. $19.5 \mathrm{~cm}$ \\
\hline
\end{tabular}


The patient had started improving during hospital stay $\&$ at end of $15^{\text {th }}$ days, there is good improvement in all symptoms of patents. After one month follow up there is nearly nil of all symptoms.

\section{Conclusion}

Ayurveda has unique concepts with all disease its chikitsa siddhanta work and stand test to the time. Since the therapy for jaodar (acitis) has limitation in other pathies, Ayurvedic management of jalodar (acitis) is one of the most effective therapy.

\section{References}

[1] Dr. Brahmanada Tripathi, Charak Samhita of Maharshi charak, Chukhambha prakashan, Varanasi, 2011, Chikitsastan, chapter 13, verse no.15, page no.472.

[2] Dr. Brahmanada Tripathi, Charak Samhita of Maharshi charak, Chukhambha prakashan, Varanasi, 2011, Chikitsastan, chapter 13, verse no.20, page no.473.

[3] Emedicine.medscape.com/article/170907-overview. (20 October 2018; 12.30 pm).

[4] Dr. Brahmanada Tripathi, Charak Samhita of Maharshi charak, Chukhambha prakashan, Varanasi, 2011, Chikitsastan, chapter 13, verse no.61, page no.483.

[5] Dr. Brahmanada Tripathi, Charak Samhita of Maharshi charak, Chukhambha prakashan, Varanasi, 2011, Chikitsastan, chapter 13, verse no.47, page no.480.

[6] Dr. Brahmanada Tripathi, Charak Samhita of Maharshi charak, Chukhambha prakashan, Varanasi, 2011, Chikitsastan, chapter 13, verse no.12-15, page no.472.

[7] Vd.vishu Gogate, Dravyagunavidnyana, Vaidyamitra prakasana, Pune, 2008, dravya no-315, page no.639.

[8] The Ayurvedic pharmacopoeia of india-part-1, Gov.of India ministry of health \& family welfare Department Of AYUSH. New Delhi; vol-2, pg.no.36.

[9] The Ayurvedic pharmacopoeia of india-part-1, Gov. Of India ministry of health \& family welfare Department of AYUSH. New Delhi; vol-1, pg.no.8.

[10] www.sharangdhar.com/ayurvedic-product/pachakvati/ (20 October 2018; $12.30 \mathrm{pm}$ ).

[11] Vd. G.P. Gune, (Aushadhi Gundharma shatra. part-2, kalpa no 10, reprint -2005, page no-208.

[12] Vd.panchanana Gune, Ayurvediya Ayushadhigunadharmashatra, Part-4, kalpa No.81, page no.453.

[13] Vd.panchanana Gune, Ayurvediya Ayushadhigunadharmashatra, Part-5, kalpa No.21, page no.661.

[14] Dr.Brahmanada Tripathi, Charak Samhita of Maharshi charak, Chukhambha prakashan, Varanasi, 2011, Chikitsastan, chapter 13, verse no.98, page no.491.

*Corresponding author.

E-mail address: dj85015@gmail.com 